\title{
A Case for an Adult Educational Technology
}

\section{Thierry Nodenot}

Laboratoire d'Informatique de l'Université de Pau et des Pays de l'Adour (LIUPPA) IUT de Bayonne, 3 avenue J. Darrigrand,64115, Bayonne, France

Thierry.Nodenot@iutbayonne.univ-pau.fr

Keywords: integration, ICT and change, innovative pedagogical methods

\begin{abstract}
The paper addresses role-oriented aspects of Open Distance Learning identifying specific components like system, co-operation and organisational facets used in the interaction-oriented learning process. We envisage the design and the operation of educational environments in which the actors (pupils, groups of pupils, instructors, ...) can interact via computerised media in the accomplishment of an activity constructed to drive learning. These interactions are educationally regulated (in an automated fashion or monitored by the teacher) from the specifications previously established by the designer of the educational activity. After presenting the principles, we describe the technological platform capable of supporting such an environment.
\end{abstract}

\section{INTRODUCTION}

Open Distance Learning (ODL) Systems have the wind in their sails. Carried by the tide of the Internet, they are pushed forward by big business in order to train their clients and colleagues and also by several universities (mainly in the United States) for whom their Web Site advertises more and more modules available on line.

In this paper, we claim a focus on educational environments based on interactions. We consider that the teacher must hold a central role even in ODL; so, rather than removing the teacher from his/her role of regulating the relationship between the learner and knowledge, we envisage a model allowing the exploitation of new Information Technologies: 
- in order to make the relationship between the learner, the knowledge and the teacher more efficient,

- in order that the teachers adhere to new technologies and profit from them at an educational level.

The suggested model works on the principal that for the teacher, a class is a system in which the interactions between actors are at least as significant as their individual performances (Tardif 1997, Mucchielli 2000, George and Leroux 2001). A detailed study of this system allows one to envisage the design (and the operation) of educational environments in which the actors (pupils, groups of pupils, instructors, teachers) are going to be able to interact via computerised media/mediums in the accomplishment of an activity constructed to drive learning. These interactions are educationally regulated (in an automated fashion or monitored by the teacher) from the specifications previously established by the designer.

After justifying our approach, we present the principals of such an environment. We end by describing the platform capable of supporting such an environment: it relies on XML, object based systems and distributed computing.

\section{OPEN DISTANCE LEARNING - INTERACTION- BASED E-LEARNING}

What is good and bad is never certain. Down the years, work and research in Computer-Aided Learning (CAL) has remained restricted to small groups of researchers. But now, as a result of new Information and Communication Technologies, we have witnessed an explosion of advertising and material for open distance learning from retailers of platforms (WebCT, Lotus Learning Space) and also from those who develop (and commercialise) educational material from this kind of platforms. Even within educational institutions, people say that very soon, on-line courses are going to compete with our lessons and for near enough same costs, the students will thus have access to individual training that is more efficient and better adapted to their needs (Lotus 1998).

Considering the challenge for our institutions, and taking into consideration the power of these firms or universities, we think it both pointless and unnecessary to fight on the same ground:

- pointless because the developers of ODL solutions have indisputable assets: a significant production capacity, a capacity to tap into a huge market-place via the Web (cheap courses but to a very large potential audience/courses at a higher cost but sufficiently in depth to interest people on a world-wide scale), a capacity to offer training on the same 
wavelength as the market and technology advance (because they are capable of mobilising, on demand, numerous human resources for the production of just in time courseware).

- unnecessary because the educational environments developed for the majority of ODL platforms on the market are very limited as to the educational dialogue they offer to the student. These are teaching aids accompanied by a methodological guide and a range of exercises allowing self-learning and self-evaluation. And this package has a userinterface with communication functions (mails, chats, videoconferencing) in order to allow the student to contact other students or an instructor during a learning session. It would be very surprising that such solutions that have been largely and justifiably criticised ten years ago for the weak educational dialogue that they provided would find public approval today only because they are circulated en masse via the web. Such solutions can probably, in the context of further education be justified but we think that other ways have to be found to ensure quality initial education aided by technology within our institutions (or in liaison with them).

We think on the other hand that it is desirable and possible to produce educational environments by using ICT which, because they are based on a model where the teacher retains a central role, will mean that these new technologies will not have the effect of removing students from our system of learning. It is therefore a question of integrating the use of new Information Technologies into every day educational practice and of finding some protocols which will entail an increase in the educational dialogue between students and teachers and consequently the educational return of our teaching (Dillenbourg 1999, Soller et al. 1999).

This education centering on interactions (Tardif 1997, Mucchielli 2000, George and Leroux 2001) that we strongly advise is complementary to education through problem-situations which are taught in teacher training colleges in France:

- the problem-situation defines the context of the interaction which is going to be established between actors (between students; between the students and instructors): it consists of presenting not only the proposed activity to the students, but also the activity objectives and problems that the students must overcome, the material they can require, the criteria to be met (both functional and formal), ...

- the study of interactions allows one to define the rules (Taveter 1999) that actors must conform to in solving the problem-situation, the modes of intervention for each actor and the dynamics of these interventions.

In earlier works, we have dealt with methods and tools for the specification of teachings based on problem-situations (Nodenot 1995, 
Marquesuzaa 1998). Here, we are going to present the characteristics of the interactive educational environment which is currently being developed in our Laboratory. It is a system of specification and regulation of co-operative activities which allows:

- The educational designer (a teacher) to define the interactions that can be established between actors (students, groups of students, human and system instructors) in the course of a problem-situation activity.

- The activities of the different actors to be monitored by the environment on the basis of the established specifications.

\section{STUDY OF A SYSTEM OF SPECIFICATION AND REGULATION OF COOPERATIVE ACTIVITIES}

This system is complex to analyse because it is an environment spread throughout space and time:

1. In space because actors whether they are students, instructors or teachers use a specific computer equipped with software dedicated to the tasks they have to achieve:

- To perform the tasks they are assigned, the learners have at their disposal tools that can be quite standard (spreadsheets, wordprocessors, programming environments, ...) or specific-purpose tools (e.g. an editor allowing the learner to know instantly the tasks that remain for him to accomplish in order to succeed). Among these tools there will also be facilities of communication which allow them to exchange information with other actors in the context of the task (the possibilities for communication are limited only to the actors with which the learner has a right to communicate in the script defined by the educational designer).

- The teachers or instructors (that intervene at the discretion of the designer) will also use dedicated tools allowing them to follow the tasks undertaken by the learners to evaluate results and to intervene in the learners activities. Our objective is to empower these actors to take decisions and to help them prioritise the need to intervene.

2. In time because all the constraints that are going to be imposed on the actors as regards the activity (via a semi-automatic regulation) are the result of an initial specification work (that is the preparation phase). At this stage, the educational designer must define the roles played by different actors, the grouping of actors, the possibilities for communication and the tools offered to different actors, the synchronisation of activities between one another with regard to information sharing and shared processes. 
To develop such an environment, we suggest an approach that would allow us to take into account all of these constraints. We explore:

- a Tool-based approach that we can summarise as such: What platform could support the activity of the user during the phases of preparation and regulation of the educational activity? What user-interfaces should be proposed to the actors to facilitate their co-operative activities?

- an Interaction-based approach to master the co-operative activities that the educational environment must promote,

- a Method-based approach whose objective is chiefly to help the designer describe the key elements of a protocol that the educational environment will enforce during the regulation phase.

We consider that these three approaches cannot be studied and implemented in a dissociated manner and incidentally, we agree with current thinking in the field of Co-operative Information Systems (an Information System is co-operative "if it shares goals with other agents in its environment, such as other information systems, human agents and the organisation itself, and contributes positively towards the fulfilment of these common goals..." (De Michelis et al. 1998)). The educational environment that we promote is a co-operative Information System that is composed of:

- A System facet whose focus is "how information will be processed and put at the disposal of actors (or at the disposal of the organisation, in general)". In our context, this facet must promote interoperability between the tools put at the disposal of the students or of the instructors whose job is to monitor the educational activity. This is based on sharing Information representing the educational specifications established during the preparation phase.

- a Co-operation facet which focuses on the actors involved in the common activity (or process) (Boloni and Marinescu 2000, Bourguin 2000, Lee et al. 2000), how they coordinate their activities to be able to adapt to unforeseen circumstances and modify their methods of work accordingly:

- An Organisational facet whose focus is to maintain consistency with regard to its educational objectives, its conditions of interaction, ... In general this facet is defined through the system specifications but in our context, such a facet must be satisfied and enforced during the regulation of the activity; consequently, this facet is largely dependent on the capacity of technology to implement and regulate such a cooperative Information system. The principal questions concerning this facet are:

1. Which description language should be used (vocabulary and level of expression of this educational language) to represent the actors, their roles in the activity, the resources they dispose to achieve their goals, the methods of co-operation that are imagined by the educational designer)? 
2. How to express the educational rules that govern the regulation of such an environment (Taveter 1999)?

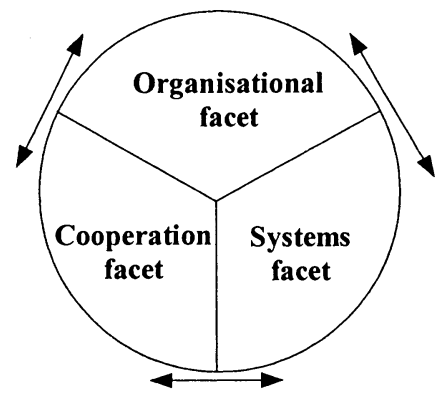

Figure 1. The three facets of an educational environment

At the moment, we try to answer both questions, from the study of the following problem-situation:

1. The educational objective for the learners is to understand the benefits of using styles with Word and to learn how to manage them,

2. The problem-situation consists of producing a Word document composed of several chapters and a table of contents. This document must be produced through the co-operation of several groups and the document must conform to a pre-defined format. These groups must coordinate their activities, exchange productions, ...

3. The obstacle that must be overcome consists of dealing with different levels of title, to associate styles with titles in a style-sheet and to generate the table of contents from these titles.

4. Three groups of students participate in this activity, helped in their tasks by the educational environment (through integrated teaching functions) and a human instructor.

5. The computerised environment is responsible for enforcing the established rules of communication,

6. The computerised environment and the human instructor if necessary can help the students in their consultation of resources (centered on formatting paragraphs, formatting styles, designing style-sheets, generating tables of contents). They must also consolidate the learning of students by undertaking consolidation exercises.

From this we highlight three facets of the educational environment. 


\subsection{Aspects of organisation/co-operation}

The objective is to provide an organisation that allows control of the interactions to be established between the different groups of learners in order to satisfy the educational objectives. The organisation specified by the designer must describe:

- the actors, their roles, their tasks

- the rules that govern the exchange of documents between actors (i.e. the document that group $\mathrm{C}$ may send to group $\mathrm{A}$ and its format, the fact that group $\mathrm{C}$ cannot modify the documents distributed by groups $\mathrm{A}$ and $\mathrm{B}$ )

- the interaction protocols between the actors (services provided by each actor, actors involved in each interaction, steps to achieve a task).

The organisation must then enforce these specifications of interaction and co-operation and this leads us to deal with the System facet.

\subsection{Aspects of organisation/system}

The educational designer must be able to describe this organisation in a declarative fashion via a simple language that is precise enough in order that, during the regulation phase, computers are able to enforce the defined rules.

Our current works are focused on the definition of such a language in order to allow a designer to express the rules of interaction between actors (information and resources that can be exchanged). These rules or Educational Partner Agreements (cf. the Trading Partner Agreements that exist to express the rules of interaction with regard to e-business trade (Sachs and Ibbotson 2000) define all the characteristics of possible interactions between actors during the educational activity (including the assistance given by the system or the human instructor). These rules can be expressed at the design level as specifications using a language like the Unified Modeling Language, or at the implementation stage as XML specifications (works in progress in our lab). We have not yet studied how to exploit these XML specifications to control the environment at the regulation stage. Yet, we know from experience that XML is well suited to automatic processing and work led on tpaML and ebxml (Grangard et al. 2000) have shown that these specifications can be used to control interactions, i.e. in e-trade environments.

\subsection{Aspects of co-operation/system}

In order to support cooperation between actors at the regulation stage, we suggest to use an Object Oriented Database Management System (OODBMS) serving the community with shared information and resources: 
- the XML specifications corresponding to EPAs defined by the designer,

- the shared educational resources which are often structured, multimedia and put at the disposal of the actors in order to aid their learning (in our example, the resources are about style sheets, generation of tables of contents, exercises for practice and consolidation).

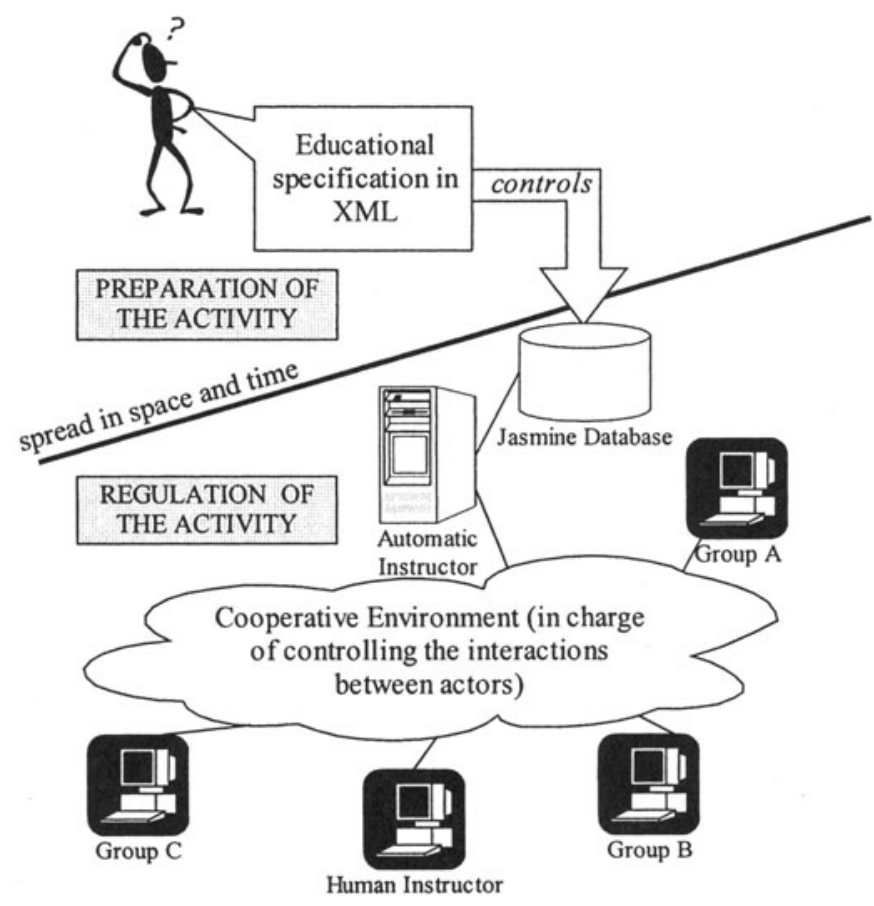

Figure 2. The topology of the system

The OODBMS that we use is Jasmine ('Computer Associates' Trade Mark). One of its advantages is to implement interfaces for different technologies (Corba, COM, ...) useful in our context. One of these interfaces is the XML provider: the XML data is thus seen through the OODBMS just like other resources stored in the database. Then, these data is accessed via a powerful query language that we exploit through Java (McCarthy 1999).

\section{CONCLUSION}

In this article, we have started to explain that there are educational and technical alternatives to the current Open Distance Learning platforms. We 
intended to show that it is possible to promote New Information Technology to enhance an educational dialogue:

- in allowing the educational designer to specify the interactions that can be established between actors (learners, groups of learners, instructors, teachers, ...) during an educational activity based on a problem-situation

- in developing a methodological infrastructure and techniques that allow the enforcement of these specifications during the interactions between actors.

In 1991, N. Balacheff questioned himself about the role of computing in education and concluded with his hope that computers could play the role of an educational partner. He wrote (Balacheff 1991): "the problem of the explanation of a tutor to the teachers, whose subject would be the nature of the apprenticeship and the types of interactions with the learner has not been, until now, studied. Yet, the pedagogues will not accept any partnership with a machine unless such a machine can communicate through a didactic language". We hope that our infrastructure will contribute, when completely implemented, to consolidate this strong idea of N. Balacheff.

\section{REFERENCES}

Balacheff, N. (1991) Contribution de la didactique et de l'épistémologie aux recherches en $E I A O$. 13 èmes journées francophones sur la Formation Intelligemment Assistée par Ordinateur (FIAO), Genève.

Boloni, D. and Marinescu, D. C. (2000) An Object-Oriented Framework for Building Collaborative Network Agents. Intelligent Systems and Interfaces. A. Kandel, K. Hoffmann, D. Mlynek, and N. H. Teodorescu (eds.), Kluewer Publishing House.

Bourguin, G. (2000) Un support informatique à l'activité coopérative fondé sur la Théorie de l'Activité: le projet DARE. Doctorat de l'Université de Lille, (nºrdre: 2753 ).

McCarthy, B. and Cassady-Dorion, L. (1999) Java Distributed Objects: the Authoritative Solution. SAMS Publishing.

De Michelis, G. et al. (1998) Cooperative Information Systems: a manifesto. In Cooperative Information Systems: Trends \& Directions, M. P. Papazoglou and G. Schlageter (eds.), Academic-Press, New York, pp. 315-363.

Dillenbourg, P. (1999) What do you mean by Collaborative Learning? Collaborative Learning: Cognitive and Computational approaches. Elsevier Science, Oxford.

George, S. and Leroux, P. (2001) Splach: Project Based Learning as a Basis for a CSCL Environment: an example in Educational Robotics. European Conference on ComputerSupported Collaborative Learning Euro-CSCL2001, Maastricht, Netherlands.

Grangard, J. A. et al. (2000) Electronic Business XML (ebXML). Technical Architecture Specification.

Lee, F. L., Liang, S. and Chan, T.-W. (2000) An attempt to design Synchronous Collaborative Learning Environments for Dyad Peers on the World Wide Web. Journal of Educational Research, vol. 21 (2), pp. 221-253.

Lotus (1998) Learning Space: solutions for anytime learning. Lotus Development Corporation Strategic White Paper. 
Marquesuzaa, C. (1998) OMAGE: Outils et Méthode pour la spécification des connaissances au sein d'un Atelier de Génie Educatif. Thèse de Doctorat de l'Université de Pau et des Pays de l'Adour ( $\mathrm{n}^{\circ}$ ordre: 412 ).

Mucchielli, R. (2000) La dynamique des groupes: processus d'influence et de changement dans la vie affective des groupes. ESF Editeur.

Nodenot, T. (1995) What if the pedagogues specified Educational Software? In Conference Proceedings WCCE'95: sixth IFIP World Conference on Computers in Education, J. D. Tinsley and T. J. van Weert (eds.), North Holland Publications, Birmingham, UK.

Sachs, M. and Ibbotson, J. (2000) Electronic Trading-Partner Agreement for E-Commerce (tpaML). Pre-submission Draft, version 1.0.3, IBM corporation.

Soller, A., Linton, F., Goodman, B. and Lesgold, A. (1999) Toward Intelligent Analysis and Support of Collaborative Learning Interaction. In Proceedings of the Ninth International Conference on Artificial Intelligence in Education, Le Mans, France.

Tardif, J. (1997) Pour un enseignement stratégique: l'apport de la psychologie cognitive. Les éditions LOGIQUES.

Taveter, K. (1999) Business Rules approach to the Modelling, Design and Implementation of Agent Oriented Information Systems. CaiSE Workshop on Agent Oriented Information Systems (AOIS'99), Heidelberg.

\section{BIOGRAPHY}

Thierry Nodenot is senior lecturer in computer science at the Institute of Technology of Bayonne (France) and responsible of a master of science in Multimedia Applications Engineering. He carries out his research at the Liuppa Laboratory in a group called Information Systems and Learning. His works dedicated to the Educational Technology focus Methods and Tools to promote and operate Problem-based (co-operative) Learning and Teaching. 